\title{
Trade-off between maximum cardinality of collision sets and accuracy of RFID reader-to-reader collision detection
}

\author{
Linchao Zhang $^{*}{ }^{\dagger}$, Filippo Gandino ${ }^{\dagger}$, Renato Ferrero ${ }^{\dagger}$, Bartolomeo Montrucchio $^{\dagger}$ and Maurizio Rebaudengo ${ }^{\dagger}$
}

\begin{abstract}
As the adoption of the radio-frequency identification (RFID) technology is increasing, many applications require a dense reader deployment. In such environments, reader-to-reader interference becomes a critical problem, so the proposal of effective anti-collision algorithms and their analysis are particularly important. Existing reader-to-reader anti-collision algorithms are typically analyzed using single interference models that consider only direct collisions. The additive interference models, which consider the sum of interferences, are more accurate but require more computational effort. The goal of this paper is to find the difference in accuracy between single and additive interference models and how many interference components should be considered in additive models. An in-depth analysis evaluates to which extent the number of the additive components in a possible collision affects the accuracy of collision detection. The results of the investigation shows that an analysis limited to direct collisions cannot reach a satisfactory accuracy, but the collisions generated by the addition of the interferences from a large number of readers do not affect significantly the detection of RFID reader-to-reader collisions.
\end{abstract}

\section{Introduction}

Radio-frequency identification (RFID) is increasingly being used in industries and infrastructures for the purpose of automatic identification and tracking [1]. An RFID system includes some RFID readers and many tags. A reader can query tags by means of a wireless communication. The majority of the RFID systems operate at ultrahigh frequency (UHF). RFID is used for many applications, such as traceability [2], item removal detection [3], anti-counterfeit [4] and positioning [5], and for the establishment of smart environments, such as smart retailers [6], smart hospitals [7], and smart universities [8]. Although the need of covering large areas has been partially satisfied by using MIMO RFID readers [9], the majority of the RFID applications, especially the largest, require a dense reader deployment, where RFID readers operate in close proximity. Consequently, UHF RFID systems are easy to suffer from the interference generated during simultaneous interrogation activities [10]. In this

\footnotetext{
*Correspondence: linchao.zhang@polito.it

${ }^{\dagger}$ Equal contributors

Dipartimento di Automatica e informatica, Politecnico di Torino, Corso Duca degli Abruzzi, 24 - 10129 Torino, Italy
}

case, a reader can suffer a reader-to-reader collision due to the interference generated by the simultaneous operations of other RFID readers [11]. When the reader queries a tag, the reader-to-reader interference is too strong with respect to the weak signals received from the tag, thus compromising the interrogation.

In recent years, many RFID reader-to-reader anticollision protocols have been proposed. The European standard for UHF RFID communication ${ }^{\mathrm{a}}$ proposes listen before talk, an anti-collision protocol based on carrier sense multiple access (CSMA). PULSE is a subsequent CSMA approach that attempts at increasing the throughput by using an additional control channel [12]. The first approach based on time division multiple access (TDMA) is Colorwave [13], which provides a simple and distributed mechanism for scheduling the query sections and is suitable for low-cost readers. More recent techniques have been proposed in order to improve the performance of Colorwave: in [14], a probabilistic parameter improves the collision resolution, and in [15], an adaptable and selfish algorithm strongly increases throughput. In the Neighbor Friendly Reader Anti-collision (NFRA) protocol

\section{Springer}

(c) 2013 Zhang et al:- licensee Springer. This is an Open Access article distributed under the terms of the Creative Commons Attribution License (http://creativecommons.org/licenses/by/2.0), which permits unrestricted use, distribution, and reproduction in any medium, provided the original work is properly cited. 
[16], a central server manages a contention among readers to schedule the query sections. NFRA provides high throughput, but it is not suitable to low-cost devices. The same technique has been enhanced in [17], where fairness among readers is improved by giving more opportunities of querying tags to the readers in the densest areas, and in [18], where the readers are scheduled in the contention phase according to the geometric distribution in order to reduce the quantity of empty time slots. Besides, the researches in $[19,20]$ provide a novel and prospective approach to limit the reader-to-reader interference by separating the transmission phase and listening phase of the RFID readers.

Although RFID reader-to-reader collisions are a relevant problem, an established method for the analysis and evaluation of reader-to-reader anti-collision protocols does not exist (e.g., [21,22]). The characteristics of the employed interference model are particularly relevant. Even considering the same deployment and the same attempts to query tags, two different models may detect different collisions. Single interference models only consider direct collisions, where the high-power transmission of a reader interferes with the low-power answers of tags to another reader. These models are easy to implement and provide rapid simulations. Additive models do not limit the analysis to direct collisions but consider the sum of the interferences from a group of readers. They are more similar to the real behavior of RFID networks but require more computational effort.

This paper investigates the characteristics of the additive interference models for detecting RFID reader-toreader collisions. In particular, the effects of the quantity of readers involved in the collisions (i.e., the cardinality of the collision set) are analyzed. The goal of the paper is to identify to which extent the collisions detected with a specific cardinality affect the accuracy of the results, in order to establish whether all the additive components must be considered for an accurate result, or instead if it is possible to limit the analysis without considering a part of the collision sets. A preliminary analysis about the interference generated by the collision sets with different cardinality has been presented in [23]. The investigation presented in the current work is based both on an analytical analysis and on simulations. The results show that an analysis of anti-collision protocols limited to direct interferences provides a low level of accuracy since many collisions are not detected. However, few collisions are due to collision sets with high cardinality, so the models used for the evaluation of RFID reader-to-reader anti-collision protocols can be limited to small collision sets.

The next section describes the state of the art of the RFID interference models. The 'Experimental setup Experimental setup' section illustrates the proposed evaluation algorithm and describes the considered scenario.
Data obtained from the evaluation are presented in the 'Experimental results' section. Final comments are made in the 'Conclusion' section.

\section{Related works}

An established dichotomy among the reader-to-reader interference models regards the cardinality of the set of colliding readers [24]. The single interference models assume that all the collisions involve pairs of readers that query tags at the same time. Given a pair of readers, it is always possible to determine if they collide or not independently of the activity of the other readers in the network. Considering the set $A$ of readers in the network, the list of pairs of readers that collide when transmitting simultaneously is a binary relation $R$ on the set $A$. Since the relation $R$ is a subset of the Cartesian product $A \times A$, it corresponds to a graph, called collision graph. The nodes of this graph are the elements of $A$, and an edge exists between two nodes $x$ and $y$ if $(x, y) \in R$. On the contrary, the additive interference models evaluate the sum of the power of all the signals received by a reader in order to determinate if a collision occurs. Considering, for example, three readers $x, y$, and $z$, according to the single interference models, if $x$ is placed far enough, it is disturbed neither by $y$ nor by $z$. However, in the additive interference models, if all the three readers query tags at the same time, the combination of the signals emitted by $y$ and $z$ can be powerful enough to interfere with the transmission of $x$. Therefore, it is not possible to build a single collision graph, i.e., to identify a priori the readers that interfere among them. Instead, for a reader $x$, a plurality of collision sets exists: each collision set groups the readers that interfere with $x$ only if all of them transmit at the same time.

In the following, the main interference models are reviewed. In the adopted notation, reader $x$ is the one that queries tags and that can be subject to reader-toreader interference from other readers in the network. The power of the signal emitted by reader $i$ is denoted as $P_{i}$. This signal propagates in the space, and it decays with distance: the signal that arrives at reader $j$ has power $P_{i, j}$, with $P_{i, j}<P_{i}$.

\section{Single interference models}

Single interference models consider only the interference between pairs of readers. The main examples of this family of models are described in the following.

\section{Disk graph model}

This model [25] assumes that the readers are equipped with an omnidirectional antenna: their signal propagates in the same way along all the directions. Due to the path loss, there is a specific distance $d$ beyond which the signal 
emitted by a reader is not powerful enough to feed the circuitry of a tag. That distance is called interrogation range: all the tags that a reader can identify are located within a circle of radius $d$, whose center is the reader itself. Formally, in the disk graph model, a reader can identify a tag if the following condition holds:

$$
|x-t| \leq d .
$$

Although outside the circle of radius $d$ the signal of the reader is too weak for querying tags, it is strong enough to disturb the simultaneous transmission of other readers. As the distance grows, the attenuation of the signal prevents the reader from disturbing the activity of the other readers: the maximum distance $D$ beyond which no interference is generated is called interference range or collision range.

In the disk graph model, each reader is characterized by constant interrogation and interference ranges. The resulting collision graph is called a disk graph [26]: an indirect edge links nodes $x$ and $y$ if $x$ is within the interference range of $y$ or, vice versa, if $y$ is within the interference range of $x$. In this case, nodes $x$ and $y$ collide when transmitting at the same time. A particular case is represented by the unit disk graph model, which assumes homogeneous readers, with the same interference range. In the unit disk graph model, the condition for avoiding interference is the following:

$$
|x-y| \geq D
$$

while in the disk graph model, the threshold $D$ is replaced by the maximum between the interference ranges $D_{x}$ and $D_{y}$ of the two readers.

In a unit disk graph, the degree of a node, i.e., the number of incident edges, corresponds to the number of readers that collide with that node in the case of a simultaneous transmission. Therefore, the degree distribution of the unit disk graph describes the discrete probability distribution of the number of distinct collisions that occur in the RFID network. This probability distribution can be calculated taking into account also the border effects of the area where readers are deployed [27].

\section{Protocol model}

According to the protocol model [28], reader $x$ can identify tag $t$ without colliding with reader $y$ if the following condition holds:

$$
|x-y| \geq(1+\Delta)|x-t|
$$

where $\Delta$ is a positive constant. The protocol model is a generalization of the unit disk graph model [24]. By assuming $D=(1+\Delta) d$, the condition for a successful tag identification in the unit disk graph model is stricter than that in the protocol model:

$$
|x-y| \geq D=(1+\Delta) d \geq(1+\Delta)|x-t|
$$

since condition 1 applies also to the protocol model, as well as in the unit disk graph model, a reader can only identify tags located within its interrogation range $d$.

\section{Capture threshold model}

In this model, the power of the signal that the reader receives from a tag is compared with the power of the interference generated by another reader that is transmitting at the same time. The comparison is repeated for all the interfering readers in the surroundings. If the ratio between the signal received by a tag and the interfering signal is higher than a threshold, then the reader identifies the tag; otherwise, a reader-to-reader collision occurs. More formally, the condition for the tag identification is the following:

$$
\frac{P_{t, x} G_{t, x}}{P_{y, x} G_{y, x}} \geq \beta_{c t}
$$

where $G_{t, x}$ is the propagation gain (including the antenna gains) from tag $t$ to reader $x$ and $G_{y, x}$ is the propagation gain from reader $y$ to reader $x$. The capture threshold model is implemented by the NS-2 simulator [29] that uses a value of $10 \mathrm{~dB}$ for $\beta_{c t}$.

The capture threshold model is a generalization of the protocol model. The two models are equivalent if the following conditions hold:

- Isotropic path loss is considered; thus, the propagation gain between points $a$ and $b$ is $G_{a, b}=\left(\frac{|a-b|}{d_{0}}\right)^{-\eta}$, where $d_{0}$ is a constant and $\eta$ is the path loss exponent.

- The readers are homogeneous: they transmit with the same power $P_{x}$ and the ratio $\frac{P_{y, x}}{P_{t, x}}$ can be considered constant.

- The value of $\Delta$ is set to $\sqrt[n]{\beta_{c t} \frac{P_{y, x}}{P_{t, x}}}-1$.

Under these hypotheses, Equation 5 can be written as

$$
\begin{aligned}
& \frac{\left(\frac{|x-t|}{d_{0}}\right)^{-\eta}}{\left(\frac{|x-y|}{d_{0}}\right)^{-\eta}} \geq \beta_{c t} \frac{P_{y, x}}{P_{t, x}} \\
& \frac{|x-y|}{|x-t|} \geq \sqrt[\eta]{\beta_{c t} \frac{P_{y, x}}{P_{t, x}}} \\
& |x-y| \geq\left(\sqrt[n]{\beta_{c t} \frac{P_{y, x}}{P_{t, x}}}-1+1\right)|x-t|=(1+\Delta)|x-t|,
\end{aligned}
$$

and the equivalence with Equation 3 is proved.

In order to correctly detect and decode the tag's reply, in the capture threshold model, it is required that the power of the received signal is higher than a threshold $\Theta$, called carrier receive level:

$$
P_{t, x} \geq \Theta .
$$


In contrast to Equation 1, condition (7) states that in the capture threshold model the interference range cannot be assumed as directly proportional to the interrogation range.

\section{Additive interference models}

The additive interference models evaluate two conditions to check if a reader can identify a tag. Firstly, the power of the signal that the reader receives from the tag must exceed the carrier receive level, as stated by condition (7). Secondly, the signal-to-interference plus noise ratio (SINR) must be higher than a required threshold $\Gamma$, which depends on the desired read rate, the bit error rate, and the kind of modulation:

$$
\frac{P_{t, x}}{I_{x}+\mathcal{N}} \geq \Gamma,
$$

where $I_{x}$ is the interference sensed by reader $x$ and $\mathcal{N}$ is the background noise power.

The main models that were proposed to evaluate Equation 8 are reviewed in the following.

\section{Single-channel model}

The model proposed in [11] considers the common situation in which the same channel is used for reader-totag query communication and for tag-to-reader response communication. The power of the signal that $\operatorname{tag} t$ receives when it is interrogated by reader $x$ is

$$
P_{x, t}=P_{x} G_{x} G_{t} \frac{P_{0}}{|x-t|^{\alpha}}
$$

where $G_{x}$ and $G_{t}$ represent the antenna gains of the reader and the tag, respectively, and $\alpha$ is the path loss exponent, whose value generally ranges from 2 to 4 . A value of $\alpha=2$ results in free space propagation, which corresponds to a line-of-sight communication between readers without interposing obstacles. $P_{0}$ is the path loss at the reference distance $d_{0}=1 \mathrm{~m} . P_{0}$ depends on the considered propagation model: in free space propagation, $P_{0}=\left(\frac{\lambda}{4 \pi}\right)^{2}$, where $\lambda$ is the signal wavelength (in meters).

Let $E_{\text {tag }}$ be the reflection coefficient of the tag antenna, i.e., the ratio of the power received by the tag that is backscattered to the reader. Then, the power received by the reader from the tag is given by

$$
P_{t, x}=E_{\mathrm{tag}} P_{x, t} G_{x} G_{t} \frac{P_{0}}{|x-t|^{\alpha}}=E_{\mathrm{tag}} P_{x}\left(G_{x} G_{t} \frac{P_{0}}{|x-t|^{\alpha}}\right)^{2} .
$$

The power of the interfering signal that reader $x$ receives from reader $y$ is calculated in the same way as Equation 9:

$$
P_{y, x}=P_{y} G_{x} G_{y} \frac{P_{0}}{|x-y|^{\alpha}} .
$$

The total interference $I_{x}$ perceived by reader $x$ is the summation of the contribution expressed by Equation 11 for all the other readers in the network. $I_{x}$ is evaluated in [11] considering the readers deployed according to the hexagonal constellation shown in Figure 1 . Reader $x$ is at the origin of the $i$ and $j$ axes. Let $L$ be the distance between two close readers; the distance between reader $x$ and a reader located at point $(i, j)$ is $L \sqrt{i^{2}+j^{2}+i j}$. With this deployment, the total interference sensed by reader $x$ is

$$
I_{x}=6 \cdot \sum_{i=0}^{\infty} \sum_{j=0}^{\infty} P_{i j} G_{x} G_{i j} \frac{P_{0}}{\left(L \sqrt{i^{2}+j^{2}+i j}\right)^{\alpha}},
$$

where $P_{i j}$ is the power of the signal transmitted by the reader located at point $(i, j)$ and $G_{i j}$ is the gain of its antenna. An upper bound is provided in the case of homogeneous readers that transmit with power $P_{x}$ and whose antenna gain is $G_{x}$ :

$$
I_{x}<6 P_{x} G_{x}^{2} P_{0} \frac{1}{L^{\alpha}} \beta\left(\frac{\alpha}{2}\right) \zeta\left(\frac{\alpha}{2}\right)
$$

where $\beta(s)$ is the Dirichlet beta function, defined as

$$
\beta(s)=\sum_{n=0}^{\infty} \frac{(-1)^{n}}{(2 n+1)^{s}}
$$

and $\zeta(s)$ is the Riemann zeta function:

$$
\zeta(s)=\sum_{n=0}^{\infty} \frac{1}{(n+1)^{s}}
$$

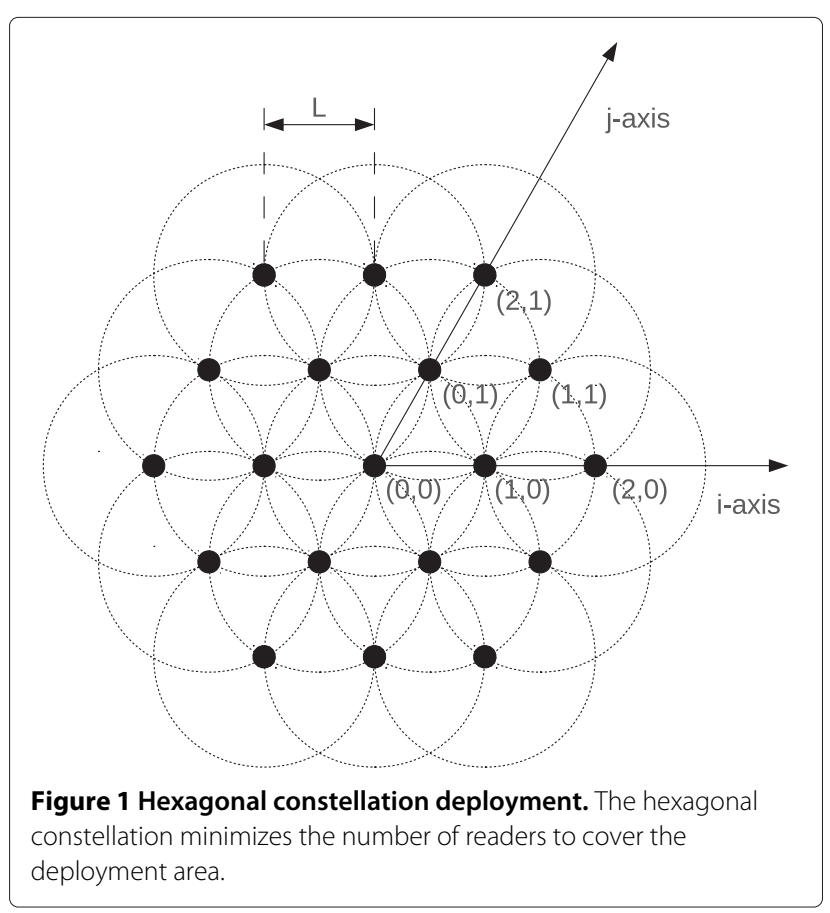


If we assume that all the RFID readers have the same antenna gain $G_{x}$ and transmitting power $P_{x}, I_{x}$ turns into

$$
I_{x}=\sum_{i=0}^{\infty} P_{x} G_{x}^{2} \frac{P_{0}}{\left|x_{i}-x\right|^{\alpha}}
$$

where $\left|x_{i}-x\right|$ is the distance between the simultaneously interfering reader $x_{i}$ and the target reader $x$.

\section{Physical model}

The physical model [28] considers the distance $L$ between the source and the destination of the signal, and it applies the proportional decay $L^{-\alpha}$ of the signal with the distance in order to evaluate Equation 8:

$$
\frac{\frac{P_{t}}{|x-t|^{\alpha}}}{\mathcal{N}+\sum_{i=0}^{N} \frac{P_{i}}{|x-i|^{\alpha}}} \geq \Gamma,
$$

where $N$ is the number of readers in the network (in addition to reader $x$ ).

The physical model is a simplification of the singlechannel model. The two models are equivalent if all the interfering readers are homogeneous with antenna gain $G_{y}=\frac{G_{x}}{P_{0}}$ and if the gain of the tag's antenna is $G_{t}=\frac{G_{x}}{P_{0} E_{\text {tag }}}$.

\section{IRRR model}

Reader-to-reader interference reduces the value of SIR measured at reader $x$ : since the power of the signal backscatterd by tags keeps constant, this reduces the interrogation range of reader $x$. The interrogation range reduction ratio (IRRR) is a parameter proposed in [30,31] to evaluate the effect of reader-to-reader interference. The power of the signal that reader $x$ receives from tag $t$ is given as

$$
\begin{aligned}
P_{t, x} & =\alpha_{\mathrm{BW}} E_{\mathrm{tag}} P_{x} G_{x} G_{t} \cdot 10^{0.2 \cdot \operatorname{PL}(|x-t|)} \\
& =\alpha_{\mathrm{BW}} E_{\mathrm{tag}} P_{x} G_{x} G_{t}\left(\frac{P_{0}}{|x-t|^{\alpha}}\right)^{2},
\end{aligned}
$$

where $\alpha_{\mathrm{BW}}$ denotes the ratio of the spectrum power in the used channel to the available bandwidth. PL is the path loss between $x$ and $t$ : since it depends on their distance, the path loss $P_{0}$ at the reference distance $d_{0}=1 \mathrm{~m}$ is adopted in the second formulation of Equation 18. The total path loss between $x$ and $t$ is obtained by summing two contributions: the first one for the forwarding readerto-tag query communication and the second one for the returning tag-to-reader response. Fading effects are ignored because a line-of-sight propagation is assumed for the reader's query and the tag's response.
The interference that $x$ receives from reader $y$ is estimated as

$$
\begin{aligned}
P_{y, x} & =h_{y} P_{y} \beta_{\text {mask }_{-}} G_{x} G_{y} \cdot 10^{0.1 \cdot \operatorname{PL}(|x-y|)} \\
& =h_{y} P_{y} \beta_{\text {mask }_{-} y} G_{x} G_{y} \frac{P_{0}}{|x-y|^{\alpha}}
\end{aligned}
$$

where $h_{y}$ is a fading coefficient in the channel between $x$ and $y$, and $\beta_{\text {mask } y}$ is the limit level of the spectrum mask.

The total interference $I_{x}$ sensed by $x$ is obtained by summing each individual contribution given by Equation 19 for all the other readers in the network. The estimation of Equation 8 easily follows. An example of evaluating equation 8 is provided in [32] by considering free space propagation, with $P_{0}=\left(\frac{\lambda}{4 \pi}\right)^{2}$, and a TDMA scheme to manage the activity of the readers. In the TDMA channel access method, the readers share the same frequency channel by allocating their transmission into different time slots. A boolean flag is introduced for each reader to indicate if it can interfere with reader $x$ :

$$
\gamma_{i}=\left\{\begin{array}{l}
1 \text { if reader } i \text { operates at the same time slot } \\
\text { of reader } x \\
0 \begin{array}{l}
\text { otherwise }
\end{array}
\end{array}\right.
$$

Under these assumptions, Equation 8 becomes

$$
\frac{\frac{\kappa_{1} P_{t, r}}{|x-t|^{4}}}{\sum_{i=1}^{N} \frac{\gamma_{i} \kappa_{2} P_{i} \beta_{\text {mask } . i}}{|x-i|^{2}}+\mathcal{N}} \geq \Gamma
$$

where $\kappa_{1}=\frac{\alpha_{\mathrm{BW}} E_{\mathrm{tag}} G_{x} G_{t} \lambda_{x}^{4}}{(4 \pi)^{4}}$ and $\kappa_{2}=\frac{h_{i} G_{x} G_{i} \lambda_{i}^{2}}{(4 \pi)^{2}}$.

An alternative way to calculate $I_{x}$ is provided in [31] by assuming a uniform random distribution of the readers. Firstly, the average interference generated by a single reader $y$ is calculated by integrating Equation 19 in the annulus where the reader $y$ can be located. $I_{x}$ is then estimated by multiplying the average interference for the average number of simultaneously active readers (given by the number of the readers in the network and their probability of querying tags).

The IRRR model extends the single-channel model by considering the availability of more than one channel for the communication among readers and tags. Furthermore, it considers fading effects in the interference among readers. The main difference between the two models lies in the estimation of $P_{t, x}$ : in the single-channel model, the contribution of the antenna gains of the reader and the tag is counted twice, while in the IRRR model, it is considered only once.

\section{Rayleigh and shadow fading model}

The interference model proposed in [33] assumes that the signals emitted by the readers randomly attenuate during their propagation according to a Rayleigh distribution. In 
addition, obstacles among the readers may further reduce the signal intensity. However, as in the other additive interference models, Rayleigh fading and shadowing can be ignored for the communication between the reader and the tag since they are in direct line of sight and within a short range. The power of the tag's reply detected by the reader is evaluated as follows:

$$
P_{t, x}=K_{1} \frac{P_{x}}{|x-t|^{4 q}},
$$

where $K_{1}$ is a constant that includes the antenna gains of the reader and the tag, the wavelength, and the modulation indexing, and $q$ models the path loss and its value depends on the environment where the signal propagates.

The interference that reader $x$ noticed from another reader $y$ is

$$
P_{y, x}=K_{2} \frac{P_{y}}{|x-y|^{2 q}} \cdot 10^{0.1 \zeta} \cdot X_{x y}^{2}
$$

where $10^{0.1 \zeta}$ takes into account the effect of shadowing and $X_{x y}$ is a random variable with Rayleigh distribution that describes the deviation in the attenuation of the signal from reader $y$ to reader $x . K_{2}$ is a constant that, similarly to $K_{1}$, considers the antenna gains of the two readers, the wavelength, and the modulation indexing.

\section{Experimental setup}

The difference between single interference and additive interference models is the cardinality $n$ of the considered collision set. Obviously, additive interference models recognize a higher number of collisions with respect to single interference models. The goal of this section is to numerically evaluate to which extent the accuracy in detecting reader interference improves as $n$ increases.

In order to avoid considering redundant collision sets, only minimal collision sets are considered in this paper. Considering a target reader $x$, the set of readers $C=$ $\left\{x_{0}, x_{1}, x_{2}, \ldots, x_{n}\right\}$ is a collision set for $x$ if the simultaneous transmissions of all the members of $C$ generate a reader interference with $x$; set $C$ is a minimal collision set for $x$ if the interference is avoided when at least one member of $C$ does not transmit at the same time as the others. Any subset of the minimal collision set cannot generate a collision; in other words, a minimal collision set does not include any other collision set. The general idea is to collect all the minimal collision sets in order to observe how many collisions can be detected by considering a collision set of cardinality at most $n$. In the rest of the paper, we refer to a minimal collision set of cardinality $n$ as collision-set- $n$.

The analysis is based on the single-channel model described in the previous section, according to the parameters listed in Table 1. A free space model is considered, assuming that no shadowing effect exists and the signal
Table 1 Evaluation parameters

\begin{tabular}{lc}
\hline Parameters & Values \\
\hline Path loss exponent $(\alpha)$ & 2 \\
SIR threshold $(\Gamma)$ & 10 \\
Reader antenna gain $\left(G_{r}\right)$ & $6 \mathrm{dBi}$ \\
Tag antenna gain $\left(G_{t}\right)$ & $1 \mathrm{dBi}$ \\
Tag's power reflection coefficient $\left(E_{\mathrm{tag}}\right)$ & $\frac{3}{4}$ \\
Reader's transmit power $\left(P_{x}\right)$ & $30 \mathrm{dBm}$ \\
Path loss at the reference distance $d_{0}\left(P_{0}\right)$ & $\frac{1}{G_{x}^{2}}$ \\
Reader-to-tag distance $(|x-t|)$ & $5 \mathrm{~m}$ \\
Noise power $(\mathcal{N})$ & 0 \\
\hline
\end{tabular}

The list of the parameters that are used in the simulations in order to compute the minimal collision sets according to the single-channel model.

power at the receiver is attenuated with a path loss exponent equal to 2 . The SIR threshold $\Gamma$ is set to 10 . All the RFID readers are considered homogeneous with an antenna gain of $6 \mathrm{dBi}$ and a constant transmit power of $10 \mathrm{dBm}$. The antenna gains of the tags are set to $1 \mathrm{dBi}$. The power reflection coefficient of the tag is $\frac{3}{4}$. $P_{0}$ is set to the upper bound $\frac{1}{G_{r}^{2}}[11]$. The distance between a reader and the queried tag $|x-t|$ is set to $5 \mathrm{~m}$. Besides, the background power is considered negligible. Furthermore, the RFID readers are assumed to be homogeneous, i.e., all the readers have the same antenna gain and transmitting power.

When the background noise power is 0 , the collision condition in Equation 8 turns into

$$
\frac{P_{t, x}}{I_{x}} \geq \Gamma .
$$

Substituting $P_{t, x}$ and $I_{x}$ according to Equations 10 and 16 , we can get

$$
\frac{P_{t, x}}{I_{x}}=\frac{E_{\mathrm{tag}} P_{x}\left(G_{x} G_{t} \frac{P_{0}}{|x-t|^{\alpha}}\right)^{2}}{\sum_{i=0}^{\infty} P_{x} G_{x}^{2} \frac{P_{0}}{\left|x_{i}-x\right|^{\alpha}}}=\frac{E_{\mathrm{tag}} G_{t}^{2} P_{0}}{|x-t|^{2 \alpha} \sum_{i=0}^{\infty} \frac{1}{\left|x_{i}-x\right|^{\alpha}}} \geq \Gamma .
$$

It can be observed that the transmitting power $P_{x}$ has no effect in the evaluation of Equation 25 for determining the occurrence of a collision, but it only affects the interrogation range. With the considered values of antenna gains and interrogation range in Table 1 , we set the transmitting power to $30 \mathrm{dBm}$ in order to make sure that the conventional RFID tags can be energized at $5 \mathrm{~m}$.

The RFID readers are randomly deployed in a $1,000 \times$ $1,000 \mathrm{~m}$ field. The position of each reader is specified in the Cartesian coordinate system, where the coordinate of each reader is randomly generated. The coordinates of all the readers are uniformly distributed and they are independent, i.e., the deployment follows a spatial Poisson 
point process. Besides, the number of readers varies from 20 to 50 in order to investigate the effect of reader densities. In order to reduce the effect of randomness, each simulation is repeated 1,000 times.

\section{Evaluation algorithm}

A branch and bound algorithm is adopted to collect all the minimal collision sets for each reader $i \in R$. The branch consists of the recursive exploration of the tree that lists all the collision sets for reader $i$. The bound is given by the identification of a minimal collision set: when this happens, all the sets that contain that collision set are discarded.

As shown in Algorithm 1, all the elements $j \in R \backslash\{i\}$ are first sorted by the descending order of the interference $P_{j i}$ received by $i$. Then a recursive procedure is called to check which are the minimal collision sets among all the subsets of $R \backslash\{i\}$. In this procedure, a stack is used to store the current subset of $R \backslash\{i\}$ in order to evaluate whether the sum of the interference of the readers in this subset can generate a collision to the target reader. When the set in the stack turns out to be a minimal collision set, all the supersets that contain the set present in the stack are ignored. Besides, a control parameter Card $_{\max }$ is introduced to

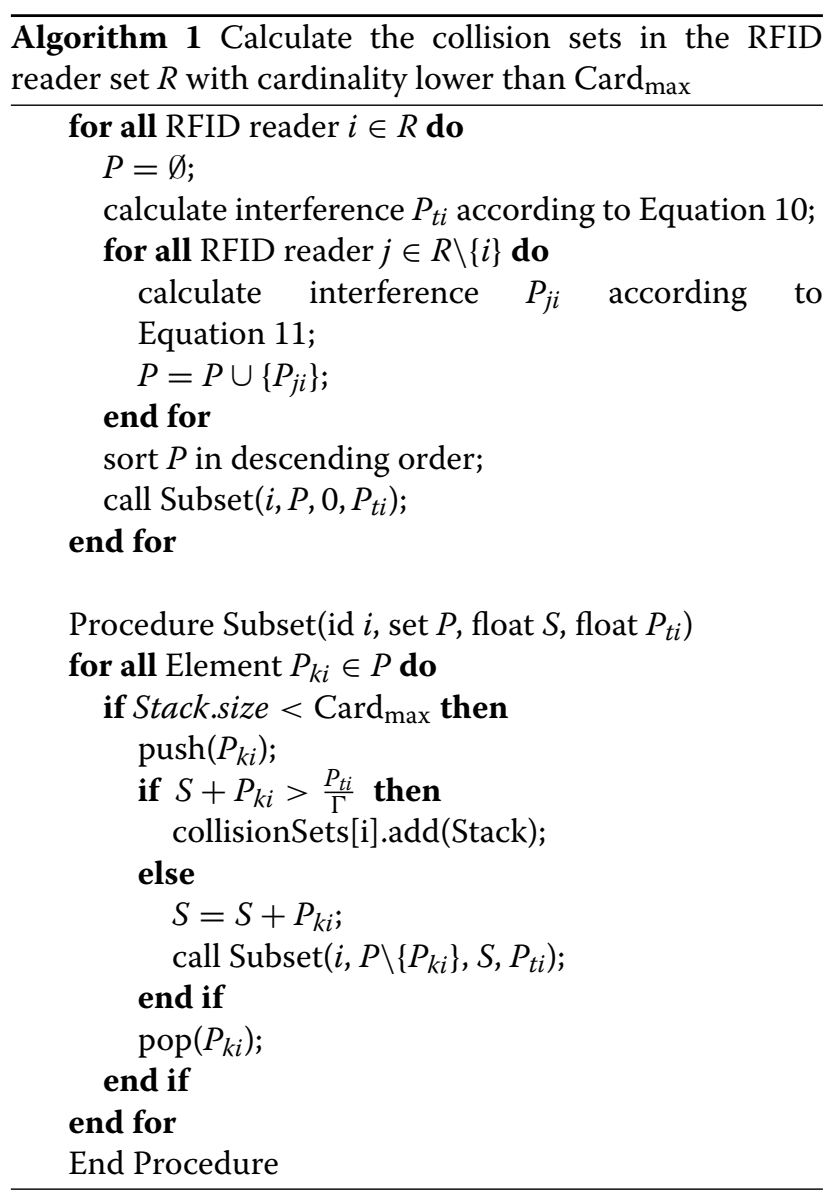

indicate that only the subsets with cardinality lower than Card $_{\max }$ are considered.

\section{Experimental results}

This section evaluates through simulation data the relationship between the cardinality of the collision set and the number of detected collisions. Different scenarios are considered by keeping a fixed field and increasing the number of readers deployed from 20 to 50 . In particular, the analysis is focused on the last scenario (i.e., 50 readers), which is used as a case study.

\section{Number of readers affected by collision-set- $n$}

If one reader has at least one collision-set- $n$, it is said to be affected by collision-set- $n$, i.e., it can experience a reader collision caused by $n$ interfering readers. Figure 2 shows the number of readers affected by different collision-set- $n$ with $n \leq 20$. The number of readers affected by collisionset- $n$ increases as the deployment density increases. It can be seen that the readers are more likely to suffer from the additive interference in a dense deployment.

Considering the scenario with 50 readers, it can be observed that almost all the readers have collision sets with cardinality from 1 to 5 . The number of readers affected by collision-set- 2 until collision-set- 5 are almost the same as the number of readers affected by direct collisions (i.e., collision-set-1), which reflects that single interference models are not enough to cover all the collisions in a dense RFID deployment. From collision-set-6, the number of affected readers starts to reduce in a great scale. The RFID reader can be affected until the sum of 14 readers' interference are considered. The simulations demonstrate that all the possible collision sets with more than 14 readers cannot generate a total interference that can hamper the target reader, which means that it is not necessary to consider additional interferences generated by more than 14 readers.

\section{Average number of collision-set- $n$}

The average number of collision-set- $n$ is the total number of collision-set- $n$ divided by the number of readers in the deployment. It can be seen in Figure 3 that the average number of collision-set- $n$ follows a Gaussian distribution in each scenario. The exact shape of the distribution depends on the characteristics of the deployment. The $x$-coordinate value of the vertex of the parabola increases from 6 to 9 when the number of readers increases from 20 to 50 . Besides, the amount of collision-set- $n$ significantly grows when the total number of readers goes up.

Under the scenario with 50 readers as shown in Figure $3 \mathrm{~d}$, the average number of collision-set- $n$ first climbs up when $n$ grows from 1 to 9 . After reaching the peak with $n=9$, it starts to fall down until $n=14$. The average number of collision-set- 15 stays at 0 , which 


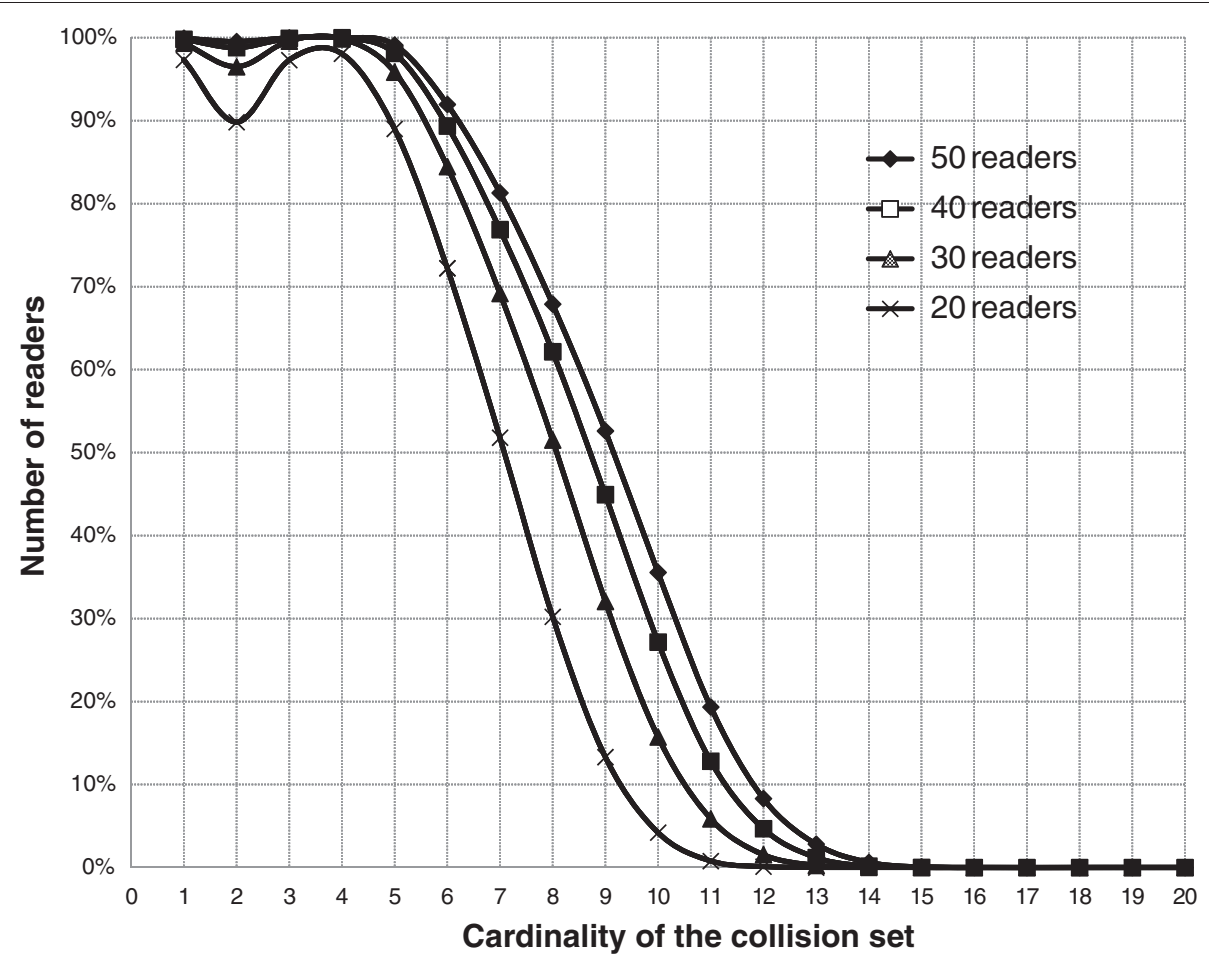

Figure 2 Number of readers affected by different collision-set- $\boldsymbol{n}$ 's. The denser the deployment is, the more readers affected by collision-set- $n$ there are.

is in accordance with Figure 2. That is because all the reader sets with cardinality higher than 14 have a subset (i.e., a minimal collision set) that already generates an interference perceived by the target reader.

When $n<9$, the average number of collision-set- $n$ climbs up because of two reasons: firstly, the probability of generating collisions increases as the number of interfering readers grows, and secondly, the number of potential subsets that may generate collisions grows with the cardinality $n$. For example, the total number of subsets with cardinality 1 is $\left(\begin{array}{l}50 \\ 1\end{array}\right)=50$, while the number of subsets with cardinality 4 grows to $\left(\begin{array}{l}50 \\ 4\end{array}\right)=230,300$. Although the maximal number of subsets continues to grow when $9<n<20$, the subsets that can generate collisions fall down since many collision sets include minimal collision sets with cardinality lower than 9 .

\section{Throughput analysis}

Considering a TDMA reader-to-reader anti-collision scheme in which each reader tries to query tags in a time slot with probability $p$, the probability that a collision set with $n$ readers generates enough noise to cause an interference is

$$
\operatorname{Pr}(\text { collision }- \text { set }-n \text { interferes })=p^{n} .
$$

Figure 4 shows how the probability that a set generates a collision exponentially decreases as $n$ increases. The value of $p$ reflects on the interrogation frequency of the RFID system. It can be observed that a low value of $p$ causes a sharper fall of the collision probability, which means that it is not necessary to consider a large cardinality $n$ of the collision set. In other words, the additive interference model seems more important in an RFID system if the readers interrogate the tags more frequently.

The average quantity of possible collisions that a reader receives at each time slot can be calculated as

$$
Q_{n}=s_{n} \cdot p^{n},
$$

where $s_{n}$ represents the average number of sets with $n$ additive components. In the case study of 50 readers deployed on a $1,000 \times 1,000 \mathrm{~m}$ square, the evaluation of (27) according to $p$ and $n$ is shown in Figure 5. It can be observed that:

- If $p$ is high, also the average number of possible collisions is high, so the probability of successfully querying tags is very low.

- If $p$ is high, the distribution curve of the quantity of collisions according to the cardinality of the collision set initially increases up to an absolute maximum and then it decreases. 


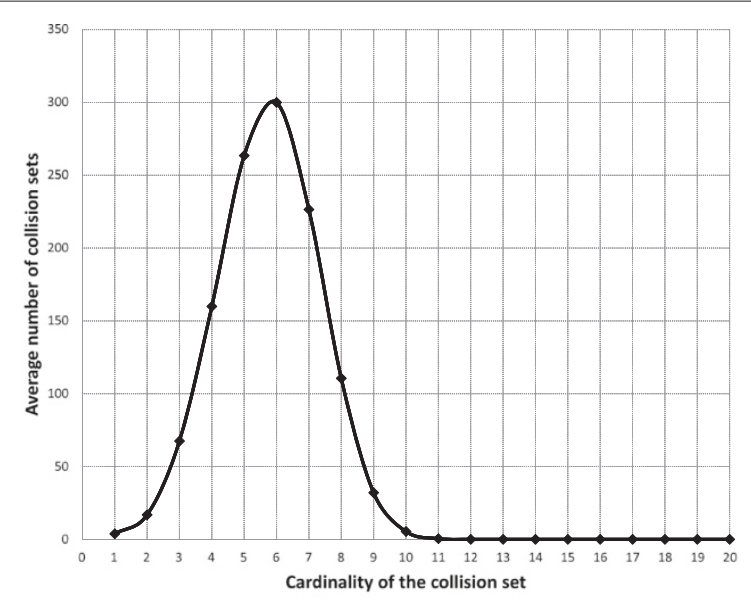

(a) 20 readers

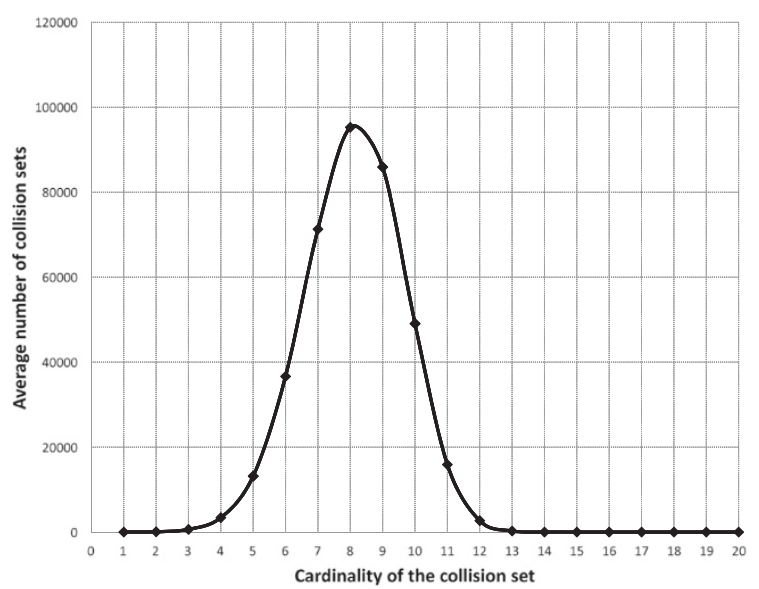

(c) 40 readers

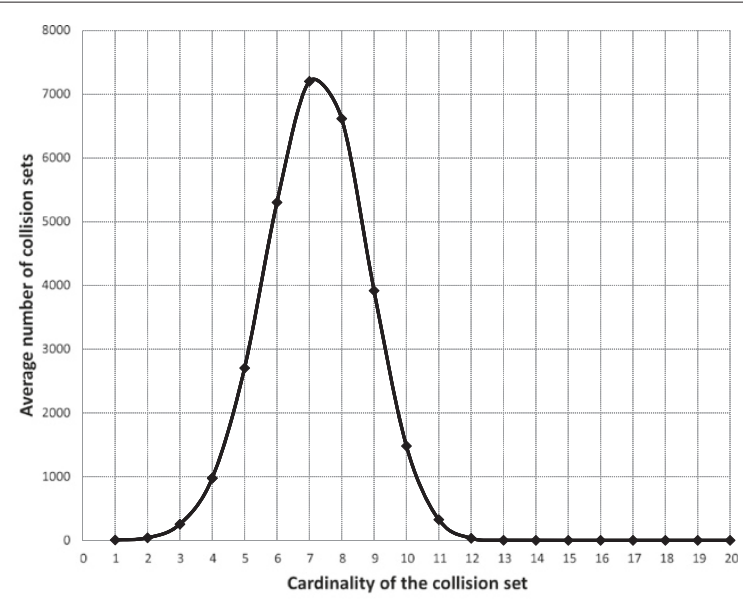

(b) 30 readers

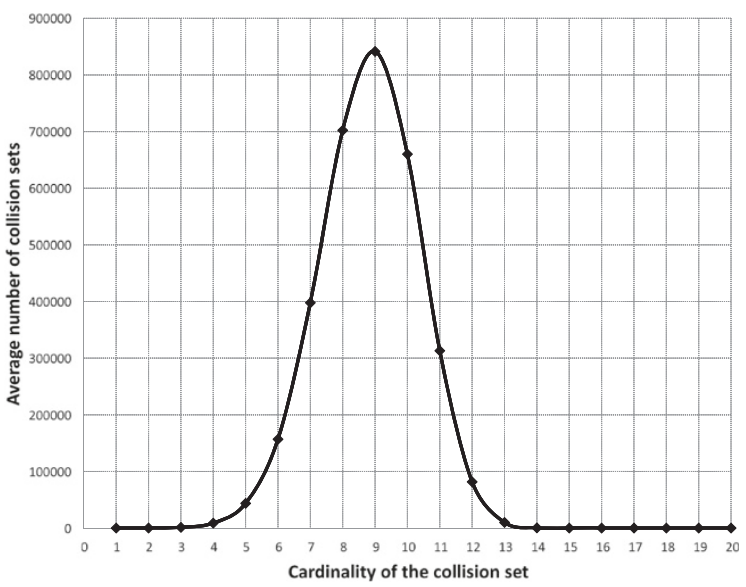

(d) 50 readers

Figure 3 Average number of collision-set-n according to different scenarios: (a) 20, (b) $\mathbf{3 0}$, (c) $\mathbf{4 0}$, (d) $\mathbf{5 0}$ readers. The average number of collision-set- $n$ follows a Gaussian distribution, whose shape depends on the characteristics of the deployment. The number of collision-set- $n$ significantly grows as the number of deployed readers increases.

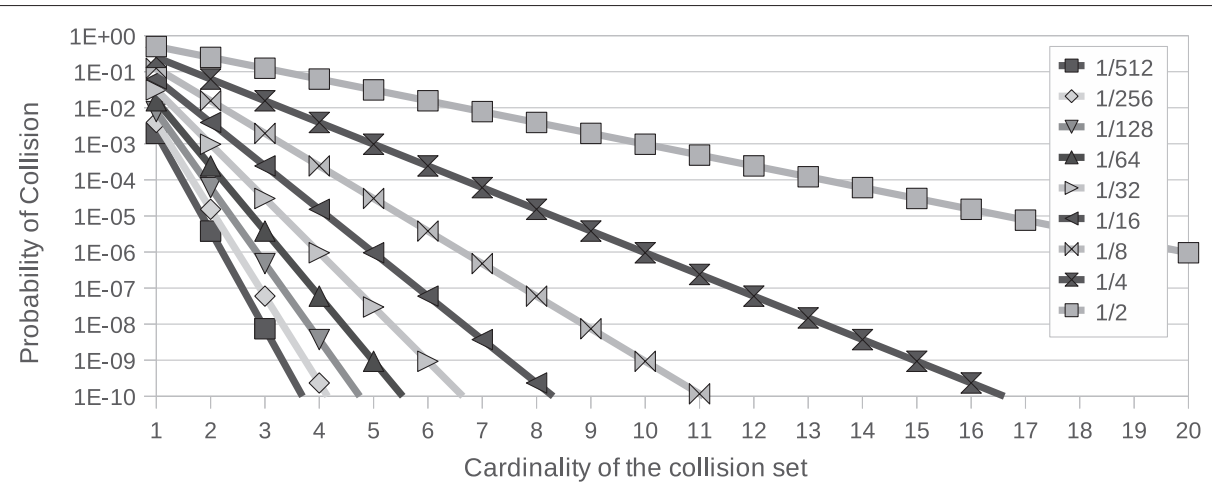

Figure 4 Probability that all the readers in collision-set- $\boldsymbol{n}$ interrogate simultaneously. The probability that a set generates a collision exponentially decreases as the cardinality of the set increases. This reduction is sharper with low values of $p$, which corresponds to lower frequency of interrogation in the RFID system. 


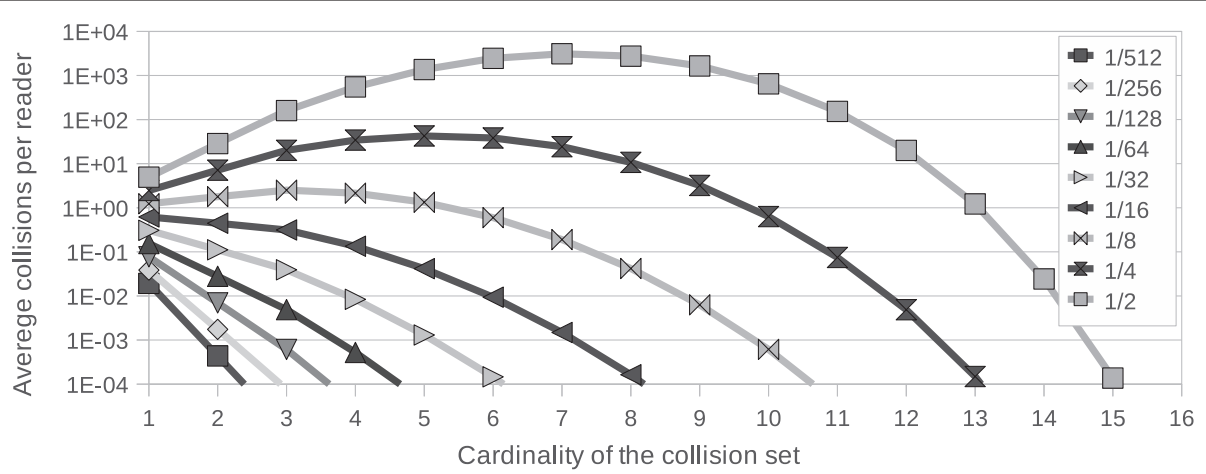

Figure 5 Average number of collision set for each reader. With a high value of $p$, the distribution of the number of collisions according to the cardinality of the collision set approaches to a Gaussian distribution. Instead, with a low value of $p$, this distribution constantly decreases and the majority of the collisions are generated by only one reader.

- If $p$ is low, the distribution curve constantly decreases and the most common collisions have only one component.

The above analysis indicates that the collision sets composed by many readers do not strongly affect the overall number of collisions. In order to further analyze the contribution of each group of collision sets, it is required to select a realistic value of $p$ that guarantees a good throughput. In this paper, the throughput is defined as

$$
T=p * p_{\text {succ }}
$$

where $p_{\text {succ }}$ represents the probability of avoiding collisions. Since the probability of avoiding a collision with a collision-set- $n$ is $1-p^{n}$, if we assume that all the $s_{n}$ collision-set- $n$ 's are independent from each other, $p_{\text {succ }}$ for $s_{n}$ collision-set- $n$ 's is $\left(1-p^{n}\right)^{s_{n}}$. Considering all the collision-set- $n$ in the specific RFID system, the overall probability $p_{\text {succ }}$ of the whole RFID system could be calculated introducing an approximation:

$$
p_{\text {succ }}=\prod_{n=1}^{N-1}\left(1-p^{n}\right)^{s_{n}} .
$$

In order to decrease the approximation, the analysis takes into account the distribution of the number of collision-set- $n$. In an RFID system with $N$ readers, the collision set with the maximum cardinality is collision-set$(N-1)$. For each value of $n$ between 1 to $N-1$, each reader has a certain number of collision-set- $n$ which is from 0 to $\left(\begin{array}{c}N-1 \\ n\end{array}\right)$. Let $d_{n}(i)$ represent the quantity of readers affected by $i$ collision-set- $n$ 's and let $X$ be the random number of collision-set- $n$, the discrete probability distribution of $X$ is characterized by the following probability mass function:

$$
\operatorname{Pr}(X=i)=\frac{d_{n}(i)}{N}
$$

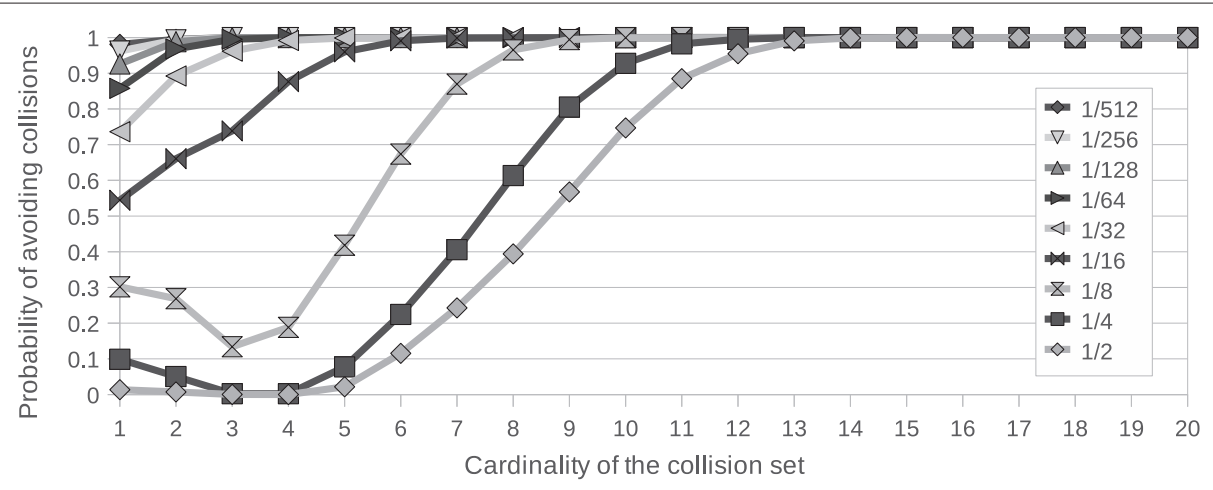

Figure 6 Probability of avoiding collisions according to the cardinality of the collision set. If $p \leq \frac{1}{16}$, the probability of avoiding collisions constantly increases as $n$ grows. Instead, if $p \geq \frac{1}{8}$, it is highly probable that a collision is generated by a set composed of 3 or 4 readers. If $n>13$, the probability of avoiding collisions is always 1 , independently of the value of $p$ is because no collision sets with cardinality higher than 13 exist. 


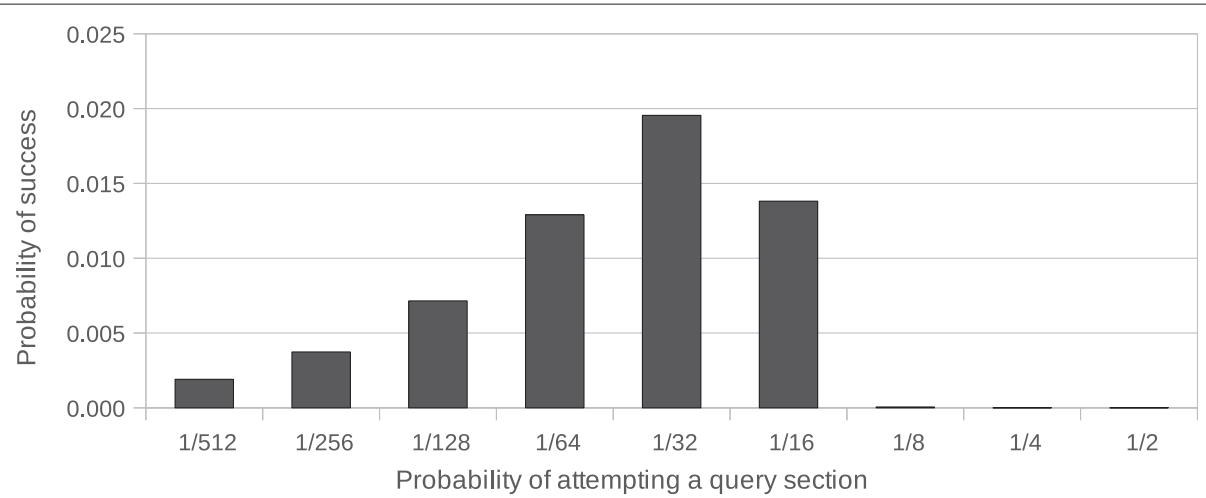

Figure 7 Throughput of the RFID system according to the probability of querying tags per time slot. The throughput is calculated according to Equations 28 and 32. The highest throughput is reached with $p=\frac{1}{32}$.

where $X \in\left[0,\left(\begin{array}{c}N-1 \\ n\end{array}\right)\right]$. Since the probability of avoiding collisions from the $i$ collision-set- $n$ is $\left(1-p^{n}\right)^{i}$, the probability to avoid collisions from all the collision-set- $n$ can be calculated as

$$
\begin{gathered}
\operatorname{Pr}(\text { no collision from collision-set- } n) \\
=\sum_{i=0}^{\left(\begin{array}{c}
N-1 \\
n
\end{array}\right)} \operatorname{Pr}(X=i) \cdot\left(1-p^{n}\right)^{1} .
\end{gathered}
$$

Figure 6 evaluates Equation 31 for different values of $p$ and $n$. It can be observed that when $p \leq \frac{1}{16}$, the probability of avoiding collisions constantly increases as $n$ grows. When $p \geq \frac{1}{8}$, it is more likely that collisions are generated by collision-set- $n$ with $n=3$ or $n=4$. Besides, when $n$ grows to more than 13, the value of $n$ will have no impact on the probability of avoiding collisions no matter what the value of $p$ is since no collision sets with cardinality higher than 13 exist.

By combining Equations 30 and 31, the overall probability of avoiding collisions in Equation 29 can be written as

$$
p_{\text {succ }}=\prod_{n=1}^{N} \sum_{i=0}^{\left(\begin{array}{c}
N-1 \\
n
\end{array}\right)} \frac{d_{n}(i) \cdot\left(1-p^{n}\right)^{i}}{N},
$$

which considers the distribution of the collision sets.

Based on Equations 28 and 32, the overall throughput of the RFID system is shown in Figure 7 with respect to different values of $p$. Since $p=\frac{1}{32}$ provides the best results, this value has been used for the subsequent analysis.

\section{Accuracy analysis}

Based on Equation 31, the probability that a collision is caused by collision-set- $n$ and that no collisions are caused by collision-set- $m$ with a smaller cardinality, i.e., $m \leq n$, is

$$
p_{n}= \begin{cases}\left(1-\sum_{i=0}^{N-1} \frac{d_{1 i}(1-p)^{i}}{N}\right), & \text { for } n=1 \\ \left(1-\sum_{i=0}^{(N-1)} \frac{d_{n i}\left(1-p^{n}\right)^{i}}{N}\right) \prod_{j=1}^{n-1} \sum_{i=0}^{\left({ }^{N-1}\right)} \frac{d_{j i}\left(1-p^{j}\right)^{i}}{N}, & \text { for } n \geq 2 .\end{cases}
$$

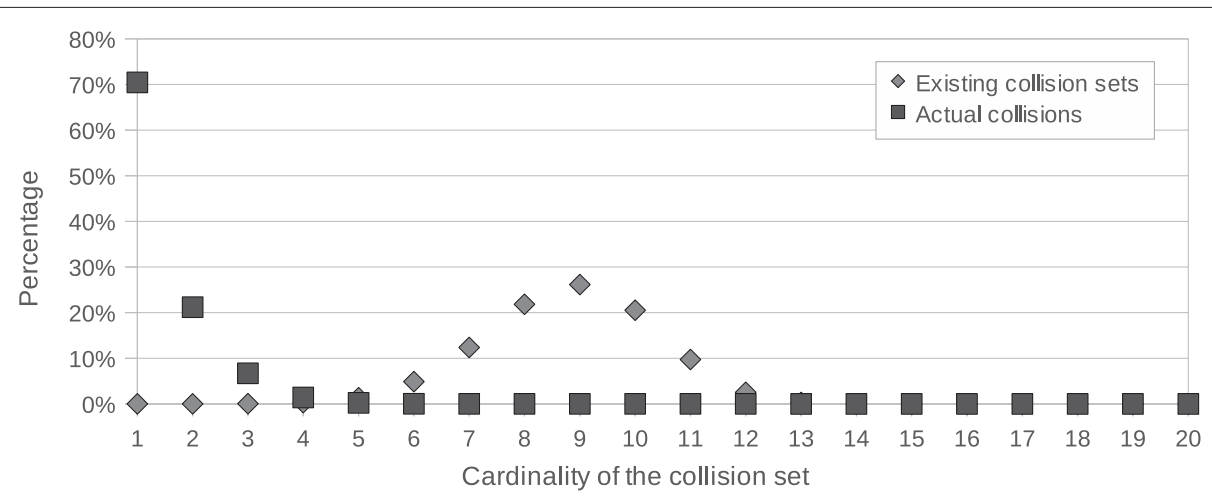

Figure 8 Distribution of possible collision sets and of actual collisions. The distribution of the collision sets approaches to a Gaussian distribution, whereas the distribution of the actual collision decreases as the cardinality of the collision set increases. Therefore, although the majority of the collision sets are composed by a number of readers comprised between 7 and 11 , the majority of the collisions are generated by sets composed by less than 3 readers. 


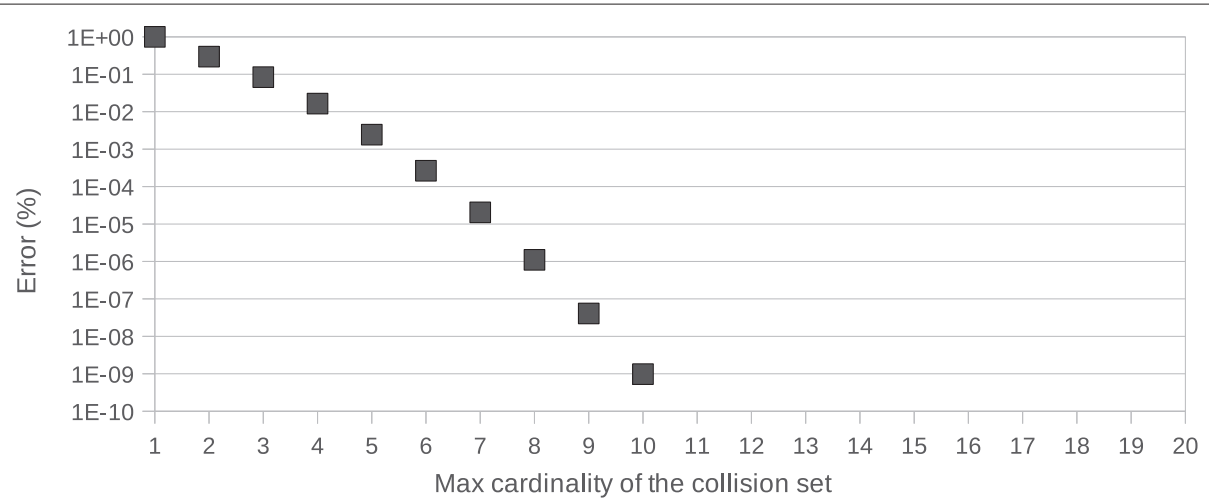

Figure 9 Accuracy of collision detection according to the maximum cardinality of the collision sets. Each point shows the error in collision detection when collision sets with cardinality larger than $n$ are not considered. The accuracy in detecting collision increases as the cardinality of the collision sets grows. When $n \geq 13$, the error is null because no collision sets with cardinality higher than 13 exist.

Figure 8 shows the distribution of potential collision sets in the network and the distribution of actual collisions, where the distribution of collision sets is based on the numerical results in Figure 3 and the distribution of actual collision probabilities is calculated as follows:

$$
D_{n}=\frac{p_{n}}{\sum_{n=1}^{N} p_{n}} .
$$

It can be observed that although the distribution of the collision sets is similar to a Gaussian distribution, the actual collision probability with cardinality $n$ decreases as the value of $n$ increases. Figure 8 also shows that the majority of the collisions are due to sets composed by few readers, while the majority of the existing sets include many readers. Therefore, a large part of the collision sets cannot be considered in the analysis of the performance of a protocol, without affecting significantly the accuracy of the results.
The percentage of error in the collision detection is shown in Figure 9. The percentage is evaluated according to the following ratio:

$$
E_{n}=1-\sum_{n=1}^{N} D_{n} .
$$

Each point in Figure 9 shows the error in collision detection when considering collision sets with cardinality up to $n$. The result shows how the accuracy of collision detection increases as the cardinality of the collision sets grows. When $n \geq 11$, the error percentage is lower than $10^{-10}$, and consequently, it is not shown because it is out of scale. Figure 10 shows the percentage of collision sets that can be covered when up to $n$ readers' interferences are summed. By comparing the two charts, it is possible to observe that the majority of the collisions can be detected even if the majority of the sets are excluded from the analysis of an RFID reader-to-reader protocol. For example, excluding all the the sets with a quantity of components larger or

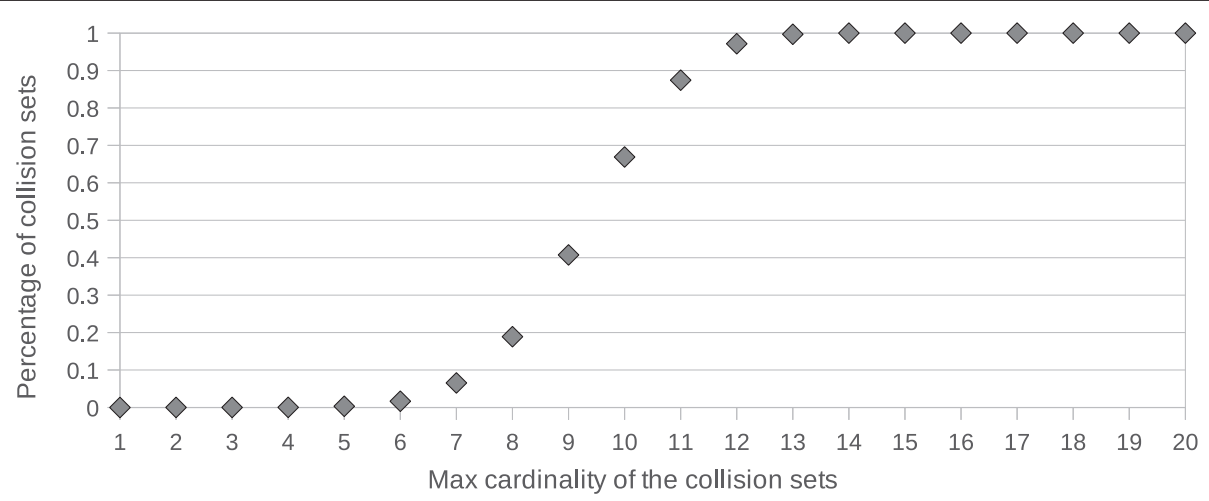

Figure 10 Percentage of detected collisions according to the maximum cardinality of the collision sets. The majority of the collisions can be detected even if the majority of the sets are excluded from the analysis of an RFID reader-to-reader protocol. 
equal to 7 , only $0.002 \%$ of the collisions are not detected, and only $6.545 \%$ of the collision sets are considered.

\section{Conclusion}

This paper has studied the characteristics of the additive interference models for detecting RFID reader-to-reader collisions. The impacts of the cardinality of the collision sets on the accuracy of collision detection has been analyzed. An evaluation based both on simulations and on an analytical analysis has shown that a model limited to direct interferences provides a low level of accuracy since many collisions could be not detected (e.g., $29 \%$ in the analyzed case study). However, a few collisions are due to collision sets with high cardinality, so the models used for the evaluation of RFID reader-to-reader anticollision protocols can be limited to small collision sets. These results open an important issue on the simulation strategies used for evaluating RFID reader-to-reader anticollision protocols and on the reliability of the simulation results.

\section{Endnote \\ ${ }^{\mathrm{a}}$ ETSI EN 300328 V1.8.1.}

\section{Competing interests}

The authors declare that they have no competing interests.

\section{Acknowledgements}

This work was supported in part by Grant 'Nano-materials and -technologies for intelligent monitoring of safety, quality and traceability in confectionery products (NAMATECH)' from Regione Piemonte, Italy.

Received: 30 November 2012 Accepted: 26 April 2013

Published: 17 May 2013

\section{References}

1. E Egea-Lopez, A Martinez-Sala, J Vales-Alonso, J Garcia-Haro, J Malgosa-Sanahuja, Wireless communications deployment in industry: a review of issues, options and technologies. Comput. Ind. 56, 29-53 (2005)

2. P Bernardi, C Demartini, F Gandino, B Montrucchio, M Rebaudengo, E Sanchez, in 21st International Conference on Advanced Information Networking and Applications, AINA. Agri-food traceability management using a RFID system with privacy protection, (Niagara Falls, 21-23 May 2007), pp. 68-75

3. M Conti, R Di Pietro, A Spognardi, "Who counterfeited my Viagra?" probabilistic item removal detection via RFID tag cooperation EURASIP. J. Wirel. Commun. Netw. 2011, 098243 (2011)

4. P Bernardi, F Gandino, F Lamberti, B Montrucchio, M Rebaudengo, E Sanchez, in 4th European Conference on Circuits and Systems for Communications, ECCSC. An anti-counterfeit mechanism for the application layer in low-cost RFID devices, (Bucharest, 10-11 July 2008), pp. 227-231

5. H Tong, J Pourrostam, SA Zekavat, LCMV Beamforming for a novel wireless local positioning system: nonstationarity and cyclostationarity analysis. EURASIP J. Adv. Signal Process. 2007, 098243 (2007)

6. JL Hou, TG Chen, An RFID-based shopping service system for retailers. Adv. Eng. Inform. 25, 103-115 (2011)

7. N Buyurgan, BC Hardgrave, J Lo, RT Walker, RFID in healthcare: a framework of uses and opportunities. Int. J. Adv. Pervasive Ubiquitous Comput. 1, 1-25 (2009)

8. M Bueno-Delgado, P Pavon-Marino, A De-Gea-Garcia, A Dolon-Garcia, in 6th International Conference on Innovative Mobile and Internet Services in
Ubiquitous Computing (IMIS). The Smart University experience: an NFC-based ubiquitous environment, (Palermo, 4-6 July 2012), pp. 799-804

9. F Zheng, T Kaiser, A space-time coding scheme for RFID MIMO systems. EURASIP J. Embedded Syst. 2012, 9 (2012)

10. M Bueno-Delgado, J Vales-Alonso, C Angerer, M Rupp, in IEEE International Conference on Industrial Technology, ICIT. A comparative study of RFID schedulers in dense reader environments, (Viña del, Mar, 14-17 Mar 2010), pp. 1373-1378

11. W Yoon, NH Vaidya, RFID reader collision problem: performance analysis and medium access. Wireless Commun. Mobile Comput. 12(5), 420-430 (2012)

12. S Birari, S lyer, in Embedded and Ubiquitous Computing, vol. 1, ed. by $T$ Enokido, L Yan, B Xiao, D Kim, Y Dai, and LT Yang. PULSE: a MAC protoco for RFID networks. LNCS (Springer, Berlin, 2005), pp. 1036-1046

13. J Waldrop, D Engels, S Sarma, in IEEE Wireless Communications and Networking, vol. 3. Colorwave: a MAC for RFID reader networks, (New Orleans, 16-20 Mar 2003), pp. 1701-1704

14. F Gandino, R Ferrero, B Montrucchio, M Rebaudengo, in 8th IEEE International Symposium on Network Computing and Applications. Introducing probability in RFID reader-to-reader anti-collision, (Cambridge, 9), pp. 250-257

15. F Gandino, R Ferrero, B Montrucchio, M Rebaudengo, DCNS: an adaptable high throughput RFID reader-to-reader anti-collision protocol. IEEE Trans. Parallel Distributed Syst. 24(5), 893-905 (2013)

16. J Eom, S Yim, T Lee, An efficient reader anticollision algorithm in dense RFID networks with mobile RFID readers. IEEE Trans. Ind. Electron. 56(7), 2326-2336 (2009)

17. R Ferrero, F Gandino, B Montrucchio, M Rebaudengo, A fair and high throughput reader-to-reader anticollision protocol in dense RFID networks. IEEE Trans. Ind. Inform. 8(3), 697-706 (2012)

18. M Bueno-Delgado, R Ferrero, F Gandino, P Pavon-Marino, M Rebaudengo, A geometric distribution reader anti-collision protocol for RFID dense reader environments. IEEE Trans. Automation Sci. Eng. 10(2), 296-306 (2013)

19. D De Donno, F Ricciato, L Catarinucci, A Coluccia, L Tarricone, in Proceedings of the 16th Annual International Conference on Mobile Computing and Networking, MobiCom '10. Challenge: towards distributed RFID sensing with software-defined radio (ACM, New York, 2010), pp. 97-104

20. D De Donno, F Ricciato, L Catarinucci, L Tarricone, in IEEE MTT-S International Microwave Symposium Digest (MTT). Design and applications of a software-defined listener for UHF RFID systems, (Baltimore, 5-10 June 2011), pp. 1-4

21. L Zhang, R Ferrero, F Gandino, M Rebaudengo, in 6th International Conference on Innovative Mobile and Internet Services in Ubiquitous Computing (IMIS). A comparison between single and additive contribution in RFID reader-to-reader interference models, (Palermo, 4-6 July 2012), pp. 177-184

22. R Verdult, $\mathrm{G}$ de Koning Gans, FD Garcia, in 4th International EURASIP Workshop on RFID Technology. A toolbox for RFID protocol analysis, (Torino, 27-28 Sept 2012), pp. 27-34

23. L Zhang, F Gandino, R Ferrero, M Rebaudengo, in 4th International EURASIP Workshop on RFID Technology. Evaluation of the additive interference model for RFID reader collision problem, (Torino, 27-28 Sept 2012), pp. 9-13

24. A lyer, C Rosenberg, A Karnik, What is the right model for wireless channel interference?. IEEE Trans. Wireless Commun. 8(5), 2662-2671 (2009)

25. C McDiarmid, Random channel assignment in the plane. Random Struct. Algorithms. 22(2), 187-212 (2003)

26. A Fishkin, in Approximation and Online Algorithms, vol. 2909, ed. by R Solis-Oba, Jansen K. Disk graphs: a short survey (Springer, Berlin, 2004), pp. 260-264. Lecture Notes in Computer Science

27. R Ferrero, F Gandino, in International Conference on Broadband and Wireless Computing, Communication and Applications (BWCCA). Degree distribution of unit disk graphs with uniformly deployed nodes on a rectangular surface, (Barcelona, 26-28 Oct 2011), pp. 255-262

28. P Gupta, P Kumar, The capacity of wireless networks. IEEE Trans. Inform. Theory. 46(2), 388-404 (2000)

29. Institute Information Sciences, ns-2 (2011). http://nsnam.isi.edu/nsnam/ index.php/Main_Page. Accessed 9 May 2013 
30. DY Kim, JG Yook, HG Yoon, BJ Jang, Interference analysis of UHF RFID systems. Prog. Electromagnetics Res. B. 4, 115-126 (2008)

31. DY Kim, HG Yoon, BJ Jang, JG Yook, Effects of reader-to-reader interference on the UHF RFID interrogation range. IEEE Trans. Ind. Electron. 56(7), 2337-2346 (2009)

32. H Seo, C Lee, in IEEE International Conference on Communications (ICC). A new GA-based resource allocation scheme for a reader-to-reader interference problem in RFID systems, (Cape Town, 23-27 May 2010), pp. 1-5

33. K Cha, S Jagannathan, D Pommerenke, Adaptive power control protocol with hardware implementation for wireless sensor and RFID reader networks. IEEE Sys. J. 1(2), 145-159 (2007)

doi:10.1186/1687-3963-2013-10

Cite this article as: Zhang et al:: Trade-off between maximum cardinality of collision sets and accuracy of RFID reader-to-reader collision detection. EURASIP Journal on Embedded Systems 2013 2013:10.

Submit your manuscript to a SpringerOpen ${ }^{\mathcal{O}}$ journal and benefit from:

- Convenient online submission

- Rigorous peer review

- Immediate publication on acceptance

- Open access: articles freely available online

- High visibility within the field

- Retaining the copyright to your article

Submit your next manuscript at $>$ springeropen.com 